\title{
UNA PINTURA Y TRES BOCETOS DESCONOCIDOS DE EDUARDO CANO
}

\section{A PAINTING AND THREE UNKNOWN SKETCHES BY EDUARDO CANO}

\author{
Álvaro Cabezas García \\ Grupo de investigación HUM317: Laraña. España \\ ORCID: 0000-0001-9675-8964 \\ alvarocabezasgarcia@gmail.com
}

\begin{abstract}
En las siguientes páginas se presentan una pintura y tres bocetos inéditos de Eduardo Cano de la Peña. Se conservan en colecciones particulares de Madrid y Sevilla y suponen nuevas versiones de otros temas recurrentes en la producción de este pintor. Todas están relacionadas con su dedicación como artista historicista, preocupado por participar de las corrientes estéticas e ideológicas de su tiempo.

Palabras clave: pintura; boceto; Eduardo Cano; siglo XIX; Historicismo.
\end{abstract}

A painting and three sketches, unpublished all of them, by Eduardo Cano de la Peña are presented in the following pages. They are kept in private collections in Madrid and Seville and are new versions of other recurring themes in the production of this painter. All of them are related to his dedication as a painter historicist, worried about participating in the aesthetic and ideological currents of his time.

Keywords: painting; sketch; Eduardo Cano; $19^{\text {th }}$ century; Historicism.

No resulta infrecuente, aún hoy, la aparición de pinturas que han permanecido inéditas para la historiografía artística, incluso de artistas de importante producción e influencia como Eduardo Cano de la Peña (Madrid, 20 de marzo de 1823 -Sevilla, 1 de abril de 1897) ${ }^{1}$. Fue considerado pintor romántico ${ }^{2}$, na-

${ }^{1}$ La monografía básica es la de PÉREZ CALERO, Gerardo: El pintor Eduardo Cano de la Peña (1823-1897). Sevilla, 1979. Algunos años más tarde FERNÁNDEZ LÓPEZ, José y VALDIVIESO, Enrique: Pintura romántica sevillana. Sevilla, 2011, pp. 240-245, publicaron interesantes fotografías de pinturas de su catálogo, que habían permanecido hasta la fecha inéditas en lo visual. También resultan interesantes los trabajos de DEL RÍO LLABONA, Armando: "La técnica pictórica de Eduardo Cano", Boletín de Bellas Artes, 25, 1997, pp. 31-42; y DE LA BANDA Y VARGAS, Antonio: "Eduardo Cano, docente y académico de Bellas Artes", Boletín de Bellas Artes, 25, 1997, pp. 43-53.

${ }^{2}$ En esta categoría lo incluye GUERRERO LOVILLO, José: "Los pintores románticos sevillanos", Archivo hispalense, XI, 1949, p. 48. Para PÉREZ CALERO, Gerardo: El 
zareno $^{3}$, impulsor de la pintura de historia en España ${ }^{4}$, aunque también hubo de practicar los géneros del retrato; participó de las corrientes costumbrista ${ }^{5}$, realista ${ }^{6}$, y ejerció como muralista, acuarelista, grabador, así como exploró el territorio del casaconismo ${ }^{7}$. Asimismo, fue un destacado defensor de la conservación del patrimonio hispalense ${ }^{8}$, de la igualdad de sexos en cuestiones de formación ${ }^{9}$ y músico amateur ${ }^{10}$.

La participación en géneros, estilos y acciones tan distintos prueban no solo que Cano fue un pintor muy activo a lo largo de una dilatada trayectoria de más de cuatro décadas, sino que además alcanzó gran consideración, no solo para sus coetáneos -al ser capaz de integrar instituciones de prestigio relacionadas con el arte y la estética ${ }^{11}-$, sino también para las generaciones posteriores, que siem-

pintor Eduardo..., op. cit., p. 67, siempre fue romántico, pero atenuado por las etapas que atravesó su trayectoria, que constantemente miró de reojo las tendencias internacionales.

3 PÉREZ CALERO, Gerardo: "Eduardo Cano, pintor romántico", en Actas del II Congreso Español de Historia del Arte. Valladolid, 1978, pp. 152-153.

${ }^{4}$ Esta faceta fue estudiada por FERNÁNDEZ LÓPEZ, José: La pintura de historia en Sevilla en el siglo XIX. Sevilla, 1985, pp. 84-87. VALDIVIESO GONZÁLEZ, Enrique: La pintura en el Museo de Bellas Artes de Sevilla. Sevilla, 1993, p. 358, dice que aunque no fue el primero que practicó pintura de historia en España, sí fue "quien realizó el primer cuadro importante con este contenido", refiriéndose al de Cristóbal Colón en el convento de la Rábida (1856, Senado, Madrid).

5 Así lo afirma QUESADA, Luis: La vida cotidiana en la pintura andaluza (cat. exp.). Sevilla, 1992, p. 120. PÉREZ CALERO, Gerardo: El pintor Eduardo..., op. cit., p. 58, había matizado que no había sido costumbrista, pero que algunos rasgos de esta práctica dejaba entrever su obra, sobre todo como consecuencia de su formación con los Bécquer.

${ }^{6}$ VALDIVIESO, Enrique: Pintura sevillana del siglo XIX. Sevilla, 1981, pp. 93-96. Idéntica afirmación hizo en VALDIVIESO, Enrique: Historia de la pintura sevillana. Siglos XIII al XX. $2^{\mathrm{a}}$ ed. Sevilla, 2002, pp. 425-426.

7 Tan solo se le conoce una pintura de este género, pero deriva claramente del estilo de Fortuny. PÉREZ CALERO, Gerardo: "Eduardo Cano y Mariano Fortuny", Archivo Español de Arte, 236, 1986, pp. 418-419.

${ }^{8}$ PÉREZ CALERO, Gerardo: "Eduardo Cano y el patrimonio artístico hispalense", en Libro homenaje al profesor Muro Orejón. Sevilla, 1979, p. 299.

${ }^{9}$ El 24 de junio de 1886 Cano defendió el establecimiento de Enseñanza para mujeres. Vid. PÉREZ CALERO, Gerardo: El pintor Eduardo..., op. cit., p. 43.

${ }^{10}$ Fue cantor intérprete ocasional y aficionado de obras vocales de Rossini. Cfr. PÉREZ CALERO, Gerardo: El pintor Eduardo..., op. cit., p. 34.

${ }^{11}$ Las corporaciones a las que perteneció fueron la Escuela de Bellas Artes de Santa Isabel de Hungría, como académico, profesor de dibujo, colorido y composición, vicedirector y director interino; miembro de la Sociedad Protectora de Bellas Artes, conservador del Museo de Bellas Artes de Sevilla y académico correspondiente de la de San Fernando de Madrid. En algunas de ellas se le tributaron reconocimientos y homenajes póstumos. El Museo de Bellas Artes de Sevilla atesora diez pinturas de Cano y el Nacional 
pre lo tomaron por maestro y referente indiscutible ${ }^{12}$. Es factible afirmar que el enorme ascendente conseguido lo debía, no tanto al número de obras que conforman su catálogo -algo menos de ciento cincuenta títulos-, o a la calidad de las mismas, sino al constante trabajo y disponibilidad que prestó como asesor, pintor y profesor, a veces de forma desinteresada y durante muchos años. Llegó a tanto su dedicación que pudo alcanzar y establecerse en la cúspide de la Escuela de pintura sevillana, personificándola -de manera inequívoca entre los años sesenta y ochenta de la centuria decimonónica-, como ningún otro artista desde el Siglo de Oro. Eso es, desde luego, lo que creían críticos como Cascales, uno de sus primeros biógrafos, que lo llamó "genio hacedor de genios" al actuar como "revitalizador" de dicha escuela, no tanto, como decía antes, por su propia pintura, sino por ser maestro del grupo de pintores que dominará el panorama artístico sevillano durante todo el primer tercio del siglo $\mathrm{XX}^{13}$. Esta posición preponderante no se refiere a una guía formal o estética para sus discípulos y alumnos al modo de $\mathrm{Mu}$ rillo -casi ninguno de ellos adeudó formas propias del maestro-, en la Escuela de Bellas Artes. Más bien se debía a la autoridad que nacía de su inexcusable

del Prado custodia una docena de obras de su mano. PÉREZ CALERO, Gerardo: El pintor Eduardo..., op. cit., pp. 21 y 48.

12 PÉREZ CALERO, Gerardo: El pintor Eduardo..., op. cit., pp. 39, 44, 134, 135 y 136 ofrece la relación de sus discípulos. Por orden cronológico fueron: María Garvey y de San Juan (1827-1918), una seguidora que hizo fruteros y retratos al modo del maestro; José Jiménez Aranda (Sevilla, 1837-1903) y José Villegas Cordero (Sevilla, 1844-Madrid, 1921), como discípulos iniciales a pesar de que cuando abandonaron Sevilla recibieron otras influencias; Virgilio Mattoni (Sevilla, 1842-1923), José García Ramos (Sevilla, 1852-1912), Emilio Sánchez Perrier (Sevilla, 1855-Alhama de Granada, 1907), José Arpa Perea (Carmona, 1858-Sevilla, 1952) y José Rico Cejudo (Sevilla, 1864-1939), lo estudiaron concienzudamente, pero no lo siguieron en lo estético. Otros fueron José Garnelo y Alda (Enguera, 1866-Montilla, 1944), Eduardo Vasallo y Doronzoro, Guillermo Álvarez Santana, Antonio Sánchez Palma (1870-1925), Manuel González Santos (Sevilla, 1875-1949), Manuel Villalobos Díaz (Sevilla, 1875-?), Diego López García (Sevilla, 1875-1969), José Pedraza Ostos (Sevilla, 1889-Valencia, 1937), y, por último, Ramona Cano (Sevilla, 1882-1952), su propia hija. Además de los anteriores, fueron favorecidos por él, entre otros, Antonio Susillo (Sevilla, 1857-1896), Rafael García y Gonzalo Bilbao (Sevilla, 1860-Madrid, 1938).

${ }^{13}$ CASCALES MUÑOZ, José: Las Bellas Artes Plásticas en Sevilla. Toledo, 1929, pp. 138-140, en las que expresa que Bartolomé Esteban Murillo fue el último genio de la escuela, y a una generación de gigantes, sucedió otra de "enanos" y copistas, ya que la escuela se convirtió en una mercantil fábrica de cuadros. Cano transformó por completo los procedimientos y el carácter de la pintura sevillana, devolviéndole los días de gloria y posicionándola donde estuvo en el pasado y le correspondía. Para él, sus cuadros fueron de menos a más, pero, en cualquier caso, todos habían conseguido venderse a altos precios. El mismo autor había escrito por primera vez sobre él en CASCALES MUÑOZ, José: Sevilla intelectual. Sevilla, 1896, pp. 305-307; y CASCALES MUÑOZ, José: "Eduardo Cano de la Peña”, en La Ilustración Española y Americana. Barcelona, 1897, p. 263. 
presencia en los círculos dictaminadores de la aprobación artística: formó parte de casi todas las comisiones que se crearon al efecto durante la segunda mitad del siglo, seleccionó los representantes de la ciudad en las exposiciones nacionales que se celebraron a partir de los años cincuenta, o los restauradores de los cuadros y el equipo encargado de elaborar -siempre bajo su dirección- los catálogos y el plan museográfico de la nueva pinacoteca sevillana. Incluso fue el representante de los pintores sevillanos ante las más altas instancias, como cuando Isabel II visitó el Museo en 1862 o cuando hizo lo mismo Alfonso XII en $1877^{14}$. Todo esto no solo lo categorizaba como prestigioso artista de conocimientos, sino que le permitía disfrutar de una selecta clientela para el desarrollo de su pintura, ya que la retrataba con asiduidad ${ }^{15}$.

Como consecuencia de lo anterior, Eduardo Cano suponía en el último tercio del siglo XIX un jalón determinante que alentaba al ejercicio de la práctica de la pintura y al comercio artístico como medio posible de vida para los jóvenes pintores. Para sus contemporáneos, no parecía haber existido una personalidad tan fuerte y poderosa desde Murillo en la escuela de pintura hispalense, ya que Cano había supuesto una frontera entre un periodo a olvidar y otro a recordar con ahínco ${ }^{16}$. Precisamente, como fuente legitimadora de la nueva etapa, protagonizada por los muchos y conocidos artistas del primer tercio del siglo XX, fue tomado por Mattoni cuando lo clasificó como "regenerador de la pintura en nuestra ciudad y maestro de una brillante pléyade de artistas"17.

${ }^{14}$ PÉREZ CALERO, Gerardo: El pintor Eduardo..., op. cit., pp. 20, 24, 25 y 33. La labor en el Museo la compartía con Joaquín Domínguez Bécquer (Sevilla, 1817-1879).

${ }_{15}$ En 1865 había conseguido retratar a la duquesa de Montpensier. Esta labor de retratista de la Sevilla oficial la heredó de José María Romero López (Sevilla, 1816-Madrid, 1894), quien la ejerció entre los años cuarenta y sesenta, antes de marchar a Cádiz y posteriormente a Madrid. A Cano, empeñado en otros quehaceres, le sucedió con este cometido Manuel Ussel de Guimbarda (La Habana, 1833-Cartagena, 1907), entre 1867 y 1886, aproximadamente, que compartió con Cano puesto en algunas comisiones, como la de la restauración de 1880 que debía ocuparse de las pinturas de la capilla del Mariscal de la catedral de Sevilla. Cfr. PÉREZ CALERO, Gerardo: El pintor Eduardo..., op. cit., p. 36.

16 PÉREZ CALERO, Gerardo: El pintor Eduardo..., op. cit., p. 117. No es de extrañar este engarce con el pasado áureo de la escuela sevillana de pintura ya que, Cano, al acometer asuntos análogos a los de los grandes maestros del pasado, como los religiosos, conseguía una acertada mezcla entre las formas de Francisco de Zurbarán (Fuente de Cantos, 1598-Madrid, 1664) y Murillo con las de Antonio María Esquivel (Sevilla, 1806-Madrid, 1857), su referente desde la distancia.

${ }^{17}$ MATTONI, Virgilio: "Sevilla en sus pintores", en AA.VV.: Quien no vio a Sevilla... Sevilla, 1920, p. 139. La misma consideración pervivió en el pensamiento de GROSSO, Alfonso: Conferencia en homenaje a la memoria del insigne pintor sevillano Gonzalo Bilbao. Sevilla, 1939, p. 8, que señalaba a Cano como el partícipe de una pintura académica, sabia y bella, que dio vida y calor a la generación de artistas de la que él provenía. 
De la misma manera que lo anterior, determinadas circunstancias vitales o rasgos de su personalidad pudieron influir favorablemente a la hora de cosechar la fama que le reportó la deferencia de ser una suerte de padre de la pintura contemporánea sevillana. De las reseñadas a través de las fuentes la primera sería el episodio artístico que estuvo a punto de costarle la vida en 1868. Cano, como destacado profesor de la Escuela de Bellas Artes y como miembro, desde 1863, de la Comisión de Monumentos Históricos y Artísticos de la Provincia de Sevilla, mantuvo una actitud pública y contraria a las acciones de derribo de murallas e inmuebles acaecidas en Sevilla entre 1867 y 1868. En la fecha en que, como consecuencia del estallido de la Revolución Gloriosa, se estaba derribando la parroquia de San Miguel, el pintor estaba allí tomando apresuradamente apuntes con los que poder ilustrar a las generaciones futuras, cuando, de improviso, cayó parte de la bóveda del edificio salvándose milagrosamente ${ }^{18}$. La segunda es el hecho que puntualiza Cascales sobre que estuvo pintando hasta sus momentos postreros, a pesar de estar casi completamente ciego ${ }^{19}$. En cualquier caso, en las décadas finales del siglo XIX, su fortuna crítica sufrió un decaimiento del que no acabó de recuperarse, a pesar de los muchos estudios emprendidos desde entonces ${ }^{20}$.

Las pinturas que aquí se estudian por primera vez reúnen todas sus características propias y están incluidas dentro del cuadro de género, de tema menor. En 1867 inauguraba Cano una temática en sus obras, que será recurrente durante sus últimos años, al participar, tanto en la exposición nacional celebrada entonces como en la respectiva de la Academia de Bellas Artes, con trece pinturas entre las que se contaban dos bocetos con los siguientes títulos: Mañara recogiendo un moribundo y El estudiante leyendo el Quijote a orillas del Manzanares ${ }^{21}$. Con estas experiencias, y en su faceta de primer pintor de la escuela sevillana, quizá pueda añadírsele a sus muchos títulos el del precursor del neobarroco ${ }^{22}$. Son, precisamente, los temas históricos o literarios de la cultura barroca -sobre todo relacionados con Cervantes o Mañara- los que practicó en mayor medida en su etapa de

${ }^{18}$ PÉREZ CALERO, Gerardo: El pintor Eduardo..., op. cit., pp. 28-29. Otras actuaciones en defensa del patrimonio sevillano fueron, también en 1868, redactar un informe sobre los conventos de religiosas que no debían ser demolidos y, al año siguiente, la defensa de la conservación del convento de San Jerónimo de Buenavista.

${ }^{19}$ CASCALES MUÑOZ, José: Las Bellas Artes..., op. cit., p. 141. Su último cuadro habría sido El testamento de Cervantes.

${ }^{20}$ Quizá el detonante de las críticas fuera SEDANO, Eugenio: Estudio de estudios. Sevilla, 1896, p. 29, que etiquetaba a Cano como un conservador que no avanzó hacia la modernidad requerida, a pesar de haber sido maestro de Villegas, un pintor que el crítico consideraba muy avanzado, y que habría recorrido el camino que el maestro dejó trazado.

${ }^{21}$ PÉREZ CALERO, Gerardo: El pintor Eduardo..., op. cit., p. 25, nota 65.

22 Ibidem, pp. 102-103, lo apuntó como precedente del taubletin y del posromanticismo al seguir, sobre todo, la pintura del Condesito de Fortuny (1861, Museo Nacional de Arte de Cataluña, Barcelona). 
madurez o ecléctica. Su interés no se limita solo al campo de la pintura, sino que también abarca el de la gestión patrimonial ${ }^{23}$. Sobre este primer tema, por consiguiente, además del notificado anteriormente, se conservan los siguientes, muy parecidos entre sí, pero con leves variaciones cada uno de ellos: Cervantes con su sobrina y un estudiante leyendo El Quijote a orillas del Manzanares (Museo de Bellas Artes, Sevilla, 1875, 17 x $25 \mathrm{~cm})$ y Un estudiante del siglo XVII leyendo El Quijote (colección de Alfonso de Vando, Sevilla, 1876, 33 x 24 cm) ${ }^{24}$.

La primera de las pinturas inéditas que presento aquí es una versión del mismo tema, pero también más tardía e interesante: Un estudiante del siglo XVII leyendo El Quijote (Figura 1). Mide 40 x $30 \mathrm{~cm}$, lleva la inscripción en rojo "E. Cano. Sa ./ 1883" en el ángulo inferior derecho y es un óleo sobre lienzo. Conservado hoy en una colección particular de Madrid, muestra solo y en medio de un paisaje muy escarpado -que permite la confusión del claro firmamento con el relieve nevado y frío de las estribaciones rocosas que se ven en lontananza- a un estudiante detenido en medio de un camino de tierra, seco y falto de vegetación. Ataviado con sombrero, viste de color pardo -en distintas tonalidades- tanto en los zapatos como en los borceguíes, el pantalón y el jubón. Solo el cuello de la camisa contrasta con un tono más claro. La capa oscura - casi caída en el suelo-, el mango de la espada y la correa completan la figura masculina que ríe con franqueza -boca entreabierta y bigotes enhiestos-, mientras lee, quizá, uno de los pasajes más entretenidos de la inmortal obra de Cervantes. La postura es de lo más desenfadada y no queda claro que el joven estudiante haya realizado un alto en el camino para leer y deleitarse en soledad o, probablemente, lo haya hecho para recitar un extracto a un auditorio situado más allá del margen del cuadro. En la versión conocida del Museo de Bellas Artes eran el propio Cervantes y su sobrina los que lo escuchaban, quizá agraciados con un sencillo homenaje tributado por un admirador de la obra del príncipe de los ingenios españoles. En cualquier caso, esta pintura es menos fina que aquella en la definición de las formas, pero más viva en la expresividad de los colores. Lo mismo podría decirse si se la compara con la versión de la colección De Vando, donde se introducía alguna decoración vegetal más y, desde luego, un acabado más estilizado a la hora de representar la anatomía y atuendo del joven estudiante, que muestra en aquella unos rasgos más

${ }^{23}$ En 1881 fue comisionado para encargarse de celebrar el bicentenario de la muerte de Calderón de la Barca y al año siguiente, bicentenario de la muerte de Murillo, hizo lo propio en relación con el pintor barroco. Vid. PÉREZ CALERO, Gerardo: El pintor Eduardo..., op. cit., pp. 37-38.

${ }^{24}$ Ibidem, p. 155. Reparó en esta actividad de Cano, VALDIVIESO, Enrique: "Temas cervantinos en la pintura sevillana decimonónica", en Discurso de ingreso del Excmo. Sr. Don Enrique Valdivieso González en la Real Academia Sevillana de Buenas Letras. Sevilla, 1996, p. 11. 
delicados -incluso a la hora de agarrar el libro-, que en esta de la colección madrileña, bastante más tardía.

Como se destacó con anterioridad, un pintor como Cano, afectado por los historicismos nacionales, quiso hacer algunas aportaciones al género en clave local, estando esta acepción muy relacionada, ya desde finales del siglo XIX, con el Barroco. Por ello, el asunto recurrente de las obras de misericordia de Miguel Mañara en beneficio de los más necesitados encontró un lecho de expansión de la mano del traductor o adaptador pictórico de su doctrina: Valdés Leal (Sevilla, 1622-1690). Ya se ha referido en alguna ocasión que, en comparación con la trascendente y determinante estética que cosechó en la pintura sevillana la obra de Murillo, la de su supuesto antagonista formal resultó bastante minoritaria ${ }^{25}$. Sin embargo, precisamente en lo más profundo de la centuria decimonónica, autores como Rodríguez de Losada (Sevilla, 1826-Jerez de la Frontera, 1896), o el propio Cano practicaron un cierto valdesianismo que utilizaban, sobre todo, como receta para la plasmación del horror o de la muerte ${ }^{26}$. Con este asunto, Cano realizó una Escena de la vida de Don Miguel de [sic] Mañara, de la que hay varias versiones. Una se encuentra en el hospital de la Santa Caridad ( 40 x $36 \mathrm{~cm}$ ); otra, en el Museo de Bellas Artes (31 x $45 \mathrm{~cm}$ ); y otra, en una colección particular de Sevilla $(32 \times 20 \mathrm{~cm})$, todas de los años sesenta. Hay otro Asunto de la vida de Don Miguel de [sic] Mañara, de 41 x $33 \mathrm{~cm}$, siendo en esta ocasión un óleo sobre tabla, firmado, pero no fechado, en la colección de Luis Pérez Lombart, de Sevilla; y, por último, Don Miguel de [sic] Mañara en uno de sus actos caritativos $(30$ x $40 \mathrm{~cm})$, que sirvió para una litografía publicada en El arte en España de $1862^{27}$.

El boceto que aquí aporto es una inédita versión del mismo tema y se debe titular Miguel Mañara visitando a un moribundo (Figura 2). Mide 29,5 x 20,5 cm, tiene la inscripción de "Boceto" y está realizado sobre tabla. Se encuentra en una colección particular sevillana y por el carácter preparatorio de la obra -además de por las dificultades de conservación que presenta- no pueden percibirse a la perfección los rasgos de los personajes o el espacio completo representado. En cualquier caso, se aprecia cómo el hermano mayor de la Caridad se acerca

${ }^{25}$ Sobre la fortuna de Murillo, vid. GARCÍA FELGUERA, María de los Santos: La fortuna de Murillo (1682-1900). Sevilla, 1989 (2a ed., Sevilla, 2017); CABEZAS GARCÍA, Álvaro: Teoría del gusto y práctica de la pintura en Sevilla (1749-1835). Sevilla, 2015; LLEÓ CAÑAL, Vicente: "Murillo en la Sevilla del siglo XIX", en NAVARRETE PRIETO, Benito (dir.): Murillo y su estela en Sevilla (cat. exp.). Sevilla, 2017, pp. 91-100; y VALDIVIESO, Enrique: La escuela de Murillo. Aportaciones al conocimiento de sus discípulos y seguidores. Sevilla, 2018. Sobre la trascendencia de Valdés, vid. LLEÓ CAÑAL, Vicente: "Fortuna crítica de Valdés Leal en el siglo XIX", en El arte del siglo XIX. II Congreso Español de Historia del Arte. T. 1. Valladolid, 1978, pp. 205-207.

${ }^{26}$ Sobre este asunto, vid. VALDIVIESO, Enrique: Valdés Leal. Sevilla, 1988.

${ }^{27}$ PÉREZ CALERO, Gerardo: El pintor Eduardo..., op. cit., pp. 154-155. 
-vestido como caballero de la orden de Calatrava- prudente y respetuoso al lecho mortuorio de un hombre que, próximo a morir, sufre los espasmos postreros que le provoca la enfermedad y la cercanía de la muerte, y cuyos síntomas se perciben en la posición de la cabeza, en la crispación de la mano derecha que agarra fuerte la manta sobre la que se recuesta, en el color macilento de la piel o en las heridas abiertas que supuran en el pecho. Una mujer, quizá la esposa del moribundo, se inclina sobre él y lamenta su suerte. Como única decoración de la lúgubre estancia, cuelga una sencilla cruz formada con palos y, en un extremo, se apoya el bastón que quizá sirviera de alivio a la carga del moribundo en los días pasados. A pesar de ser un boceto, Cano introduce aquí algunos detalles de enorme calidad, como el inteligente uso de la luz, que cae sobre la cruz de la cabecera del lecho por un lado y, por otro, sobre la figura de Mañara, que proyecta una casi fantasmagórica sombra sobre la pared, y que a pesar de la impresión que muestra ante la presencia de la muerte -su mano izquierda expresa horror junto a la espada, mientras la derecha toca su pecho-, está señalado por esa luz como nuevo salvador de los pobres y moribundos, consciente de su misión de enterrar a los muertos siguiendo las obras de misericordia divinas.

Siguiendo el mismo tema del gusto por representar lo trágico y realista, el momento postrero o el tránsito hacia la muerte, Cano realizó varias obras como Velatorio, de 69 × $51 \mathrm{~cm}$, un óleo sobre lienzo propiedad de Manuel Piñanes de Tena, de Sevilla, y firmado en $1889^{28}$. Posiblemente, como una variación del mismo tema debe entenderse Moribundo (Figura 3), de 28,5 x 20,5 cm y conservado en la misma colección sevillana que el anterior. También con la inscripción "Boceto" en el ángulo inferior izquierdo, y pintado sobre tabla, Cano origina aquí una estancia de patente pobreza e insalubridad -muros manchados por la verdina, telones raídos para separar los cubículos-, donde un hombre acaba de morir o está próximo a hacerlo sobre un lecho formado por una esterilla, una almohada, sábana y manta de color grana. El varón, que muestra su torso mortecino y el brazo derecho laxo, aparece barbado y con una compresa dispuesta sobre la frente por la anegada esposa que, sedente junto a él, llora su pérdida -enjugándose el rostro con un destacado pañuelo blanco sobre el oscuro atuendo-, y la de la hija de ambos, de uno o dos años de edad, que juega ajena al drama, junto al lecho de su padre, con una pelotita de trapo. De nuevo Cano intenta aquí representar, con detallismo, aquellos rasgos de extremo naturalismo vigentes en la literatura de la época que abogaban por mostrar la cara más amarga de la sociedad decimonónica.

${ }^{28}$ Ibidem, p. 163, cree que podría ser Un pobre, una obra desaparecida, pero citada por José Pinelo con el número 23 de su Catálogo de la XVII Exposición de Pintura española celebrada el 18 de julio de 1921 en Buenos Aires. 
Por último, hay otro asunto sobre el que Cano vuelve de modo recurrente: el de la representación de las Santas Justa y Rufina encarceladas ${ }^{29}$. La versión más conocida del tema mide $37 \times 26 \mathrm{~cm}$, es un óleo sobre tabla y se encuentra en una colección particular de Sevilla firmado, pero con fecha ilegible. Otra, pero de mayores dimensiones $(286$ x $200 \mathrm{~cm})$, y en esta ocasión óleo sobre lienzo, se encuentra en una colección particular de Málaga, pero sin firmar ni datar. El último boceto que aquí presento, de la misma colección particular sevillana de los anteriores, podría ser el preparatorio de las obras mencionadas. Con el mismo título, Santas Justa y Rufina encarceladas (Figura 4), mide 33 x $22 \mathrm{~cm}$, está realizado sobre tabla y se encuentra firmado como "E. Cano" en el extremo inferior derecho. Con importantes craquelados a ambos lados, se presentan las dos santas en el interior de una celda donde no hay nada: solo algunos roedores parecen retozar en la paja y la inmundicia del suelo. Justa, la hermana mayor, de cabello negro y ataviada con una túnica blanca y un manto verde, reconforta de rodillas a Rufina, derrumbada a sus pies y ataviada con una túnica parecida y con manto ocre. Quizá hayan sufrido ya algunos de los martirios a las que fueron sometidas por su oposición a la autoridad hispanorromana, tal y como demuestran sus ropas deshilachadas y los rostros compungidos que imploran a Dios elevándose al cielo. Desde luego fueron muy frecuentes las representaciones de las copatronas de Sevilla a lo largo de la historia de la pintura hispalense, pero la forma tradicional que se tuvo de hacerlo era como sostenedoras de la Giralda en el terremoto de 1504 , pero casi nunca como presas y reas de muerte en lo más profundo y oscuro de las santas cárceles. Esta transgresión la cometieron los referentes sevillanos de Cano en Madrid: Antonio Esquivel (Santas Justa y Rufina, 1844, Museo Nacional del Romanticismo, Madrid) y Gutiérrez de la Vega (Sevilla, 1781-Madrid, 1865) (Santa Justa y Santa Rufina, 1846, colecciones reales, Patrimonio Nacional, Madrid $)^{30}$. En la segunda mitad de la centuria, Cano desarrolló esta opción aun con mayor dramatismo y obstinación gracias a bocetos como este, en el que, si no fuera por la perspicaz iluminación, las figuras pasarían desapercibidas en su carácter santo.

${ }^{29}$ Registrado en el catálogo de PÉREZ CALERO, Gerardo: El pintor Eduardo..., op. cit., p. 152.

${ }_{30}$ Ambas pinturas fueron estudiadas por NAVARRO, Carlos G.: "60. Antonio María Esquivel y Suárez de Urbina (Sevilla, 1806-Madrid, 1857). Santas Justa y Rufina” y "61. José Gutiérrez de la Vega y Bocanegra (Sevilla, 1781-Madrid, 1865). Santa Justa y Santa Rufina", en NAVARRETE PRIETO, Benito (dir.): Murillo y su estela..., op. cit., pp. 288293. 
Cano, el pintor proveniente de una familia de arquitectos ${ }^{31}$, empezó copiando a Murillo, como era obligado en la Sevilla del siglo XIX ${ }^{32}$, siguió trabajando en la ciudad dominada por los Bejarano y los hermanos José y Joaquín Domínguez Bécquer, en el Madrid de los Madrazo $^{33}$ y hasta en el París de Courbet ${ }^{34}$, hasta acabar por ser el mejor definidor de la pintura de historia en España ${ }^{35}$, ensayando géneros y modelos tan variados que sirvieron de ejemplo a otros pintores e impregnaron de un determinado sello el panorama artístico local, al menos hasta el estallido de la Guerra Civil (1936-1939). La pintura y los bocetos que aquí se presentan por primera vez contribuyen a incrementar el conocimiento de su obra artística.

Fecha de recepción: 30 de octubre de 2018

Fecha de aceptación: 1 de julio de 2019

${ }^{31} \mathrm{Su}$ padre fue el arquitecto municipal de Sevilla Melchor Cano (1794-1842), desde 1826 hasta su fallecimiento. PÉREZ CALERO, Gerardo: El pintor Eduardo..., op. cit., p. 13.

32 En mayo de 1849 Cano realiza copias de las pinturas Concepción, Anunciación y Nacimiento, que, provenientes de la iglesia del convento de capuchinos, estaban desde la reciente desamortización en el Museo de Pinturas de Sevilla. Cfr. PÉREZ CALERO, Gerardo: El pintor Eduardo..., op. cit., p. 15.

${ }_{33}$ PÉREZ CALERO, Gerardo: El pintor Eduardo..., op. cit., p. 60.

${ }_{34}$ Allí coincidió con Benito Soriano Murillo (Palma de Mallorca, 1827-Madrid, 1891), Martín Tovar (Caracas, 1827-1902), José Salomé Pina (Ciudad de México, 18301909) y Gabriel Maureta (Barcelona, 1832-Madrid, 1912). Cfr. PÉREZ CALERO, Gerardo: El pintor Eduardo..., op. cit., p. 17.

35 PÉREZ CALERO, Gerardo: El pintor Eduardo..., op. cit., p. 71. 
Figura 1. Eduardo Cano de la Peña, Un estudiante del siglo XVII leyendo El Quijote, 1883, colección particular, Madrid.
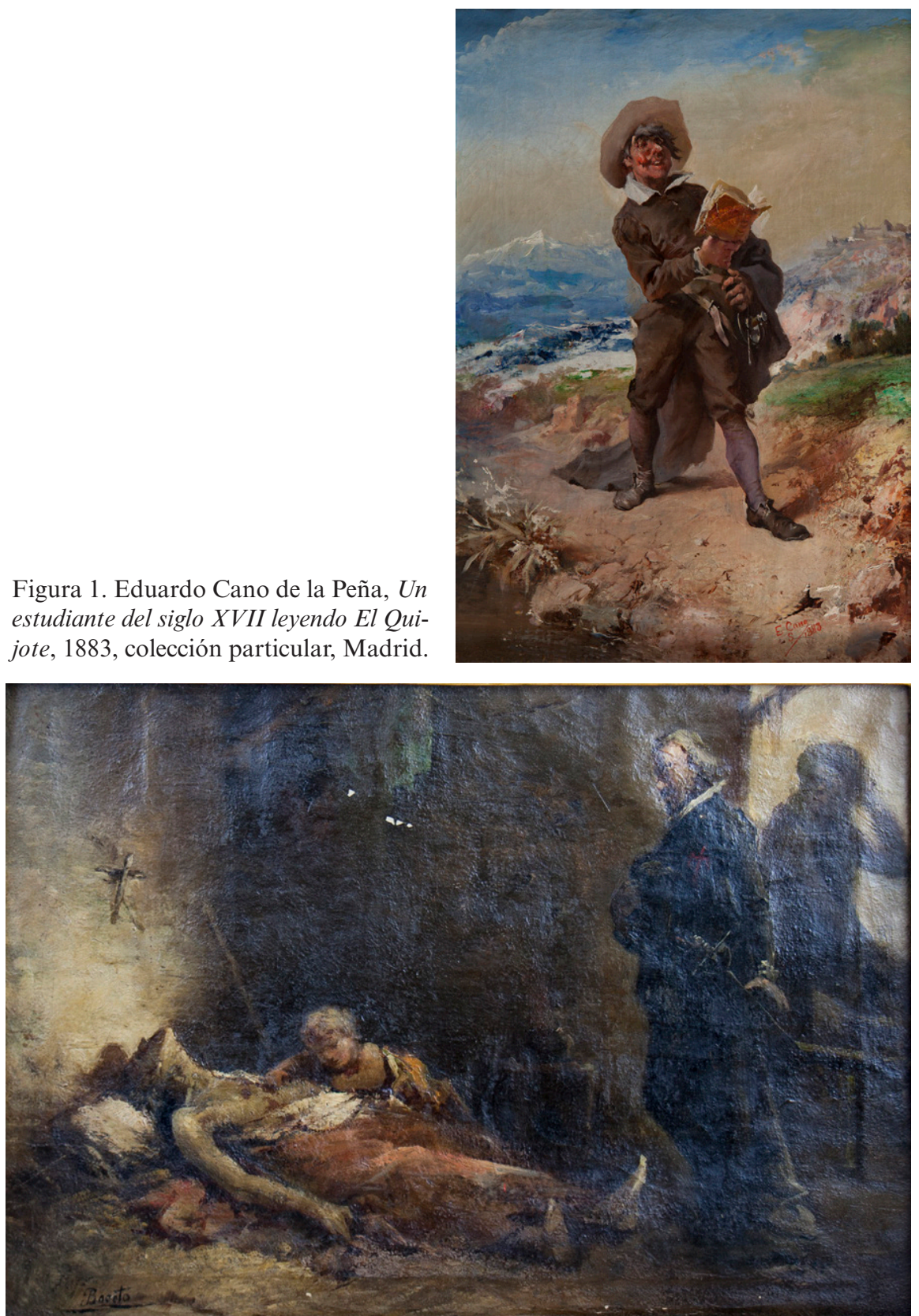

Figura 2. Eduardo Cano de la Peña, Miguel Mañara visitando a un moribundo, colección particular, Sevilla. 


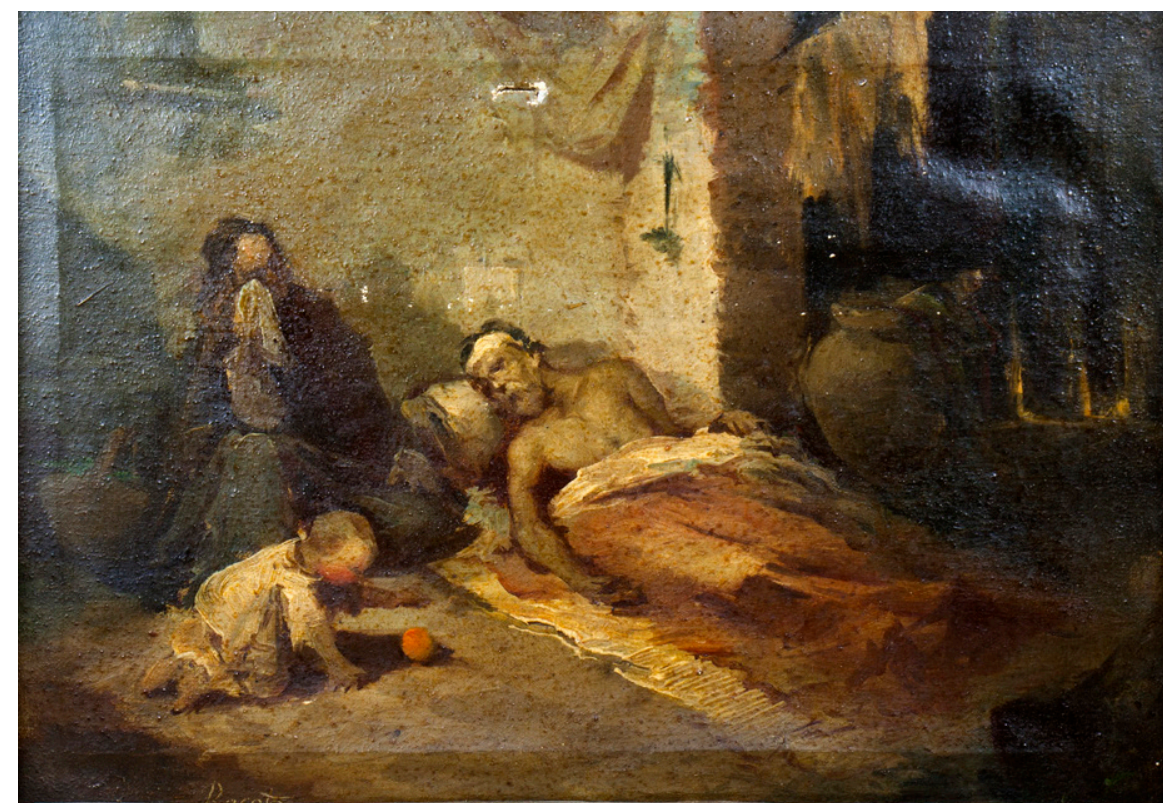

Figura 3. Eduardo Cano de la Peña, Moribundo, colección particular, Sevilla.

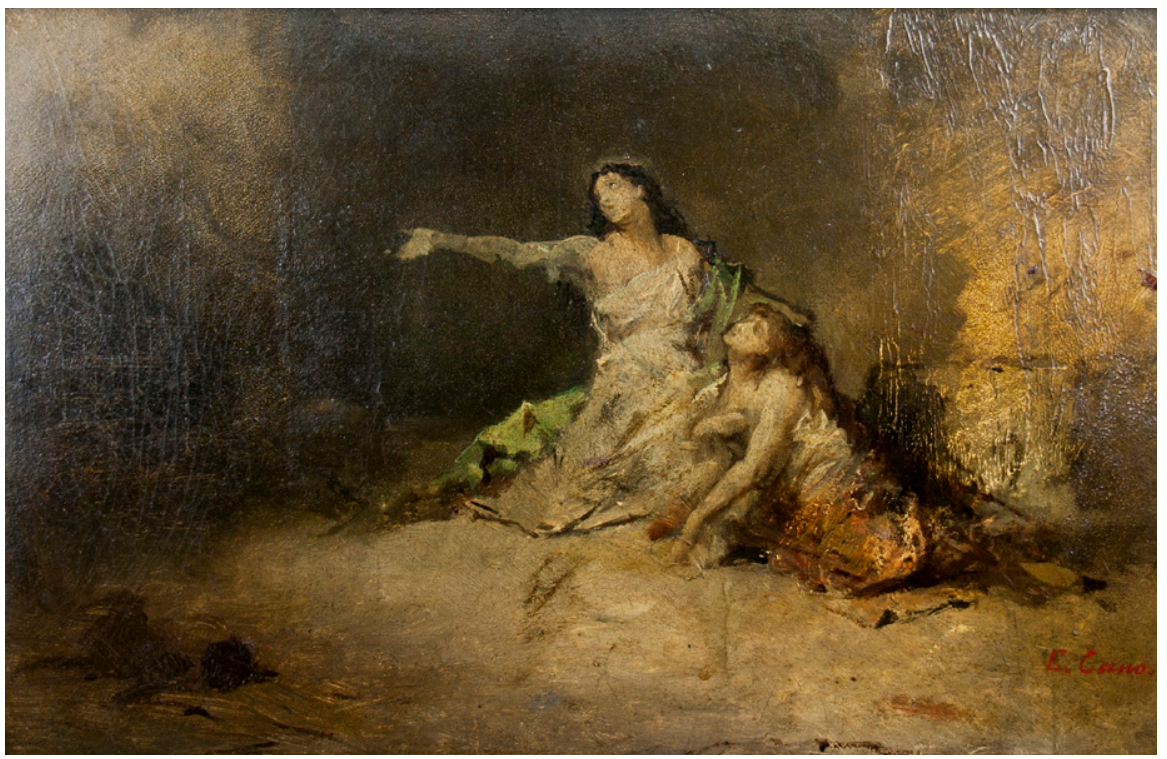

Figura 4. Eduardo Cano de la Peña, Santas Justa y Rufina encarceladas, colección particular, Sevilla. 